\title{
Second primary malignancies associated with radiation therapy in cervical cancer patients diagnosed between 1975 and 2011: a population-based competing-risk study
}

\author{
Yijun $\mathrm{Wu}^{1,2 \#}$, Yuming Chong ${ }^{2 \#}$, Chang Han ${ }^{2 \#}$, Kai Kang ${ }^{1,2}$, Zhikai Liu ${ }^{1}$, Fuquan Zhang ${ }^{1}$ \\ ${ }^{1}$ Department of Radiation Oncology, Peking Union Medical College Hospital, Chinese Academy of Medical Sciences \& Peking Union Medical \\ College, Beijing, China; ${ }^{2}$ Peking Union Medical College, Eight-year MD program, Chinese Academy of Medical Sciences, Beijing, China \\ Contributions: (I) Conception and design: Y Wu, C Han; (II) Administrative support: Z Liu, F Zhang; (III) Provision of study materials or patients: \\ C Han, K Kang; (IV) Collection and assembly of data: C Han, Y Chong; (V) Data analysis and interpretation: Y Wu, Y Chong; (VI) Manuscript \\ writing: All authors; (VII) Final approval of manuscript: All authors. \\ \#These authors contributed equally to this work. \\ Correspondence to: Zhikai Liu, MD; Fuquan Zhang, MD. Department of Radiation Oncology, Peking Union Medical College Hospital, Chinese \\ Academy of Medical Sciences \& Peking Union Medical College, Beijing, China. Email: liuzk2009@126.com; zhangfq_pumc@163.com.
}

Background: Cervical cancer is a major health threat for women. Radiotherapy plays an important role in the treatment of cervical cancer. However, its overall benefit has been questioned due to the risk of second primary malignancies.

Methods: The Surveillance, Epidemiology, and End Results (SEER) database was used to search for cervical cancer patients diagnosed between January 1975 and November 2011. Factors that could possibly affect the occurrence of second primary malignancies included the year of diagnosis, gender, ethnicity, histologic type, SEER cancer stage, histology, grade, and whether surgery, chemotherapy, or radiotherapy were used. Age-adjusted and propensity scoring matching (PSM)_adjusted competing-risk analysis was applied for analysis.

Results: Of the 23,112 patients identified through SEER, 14,800 (64.0\%) received radiotherapy. Second malignancies were diagnosed in 2,545 (11.0\%) cases. PSM-adjusted competing analysis revealed that patients receiving radiotherapy had a significantly higher risk of developing a second cancer in the colon, rectum and anus [hazard ratio (HR): 1.43; 95\% confidence interval (CI): 1.09-1.87; $\mathrm{P}=0.01$ ], lung and bronchus (HR: 1.41; 95\% CI: 1.13-1.76; $\mathrm{P}=0.002$ ), corpus uteri (HR: 3.71; 95\% CI: 1.71-8.06; $\mathrm{P}<0.001$ ), ovary (HR: 2.79; 95\% CI: 1.38-5.64; P=0.004), and urinary bladder (HR: 2.18; 95\% CI: 1.35-3.54; $\mathrm{P}=0.002$ ). However, radiotherapy significantly lowered the risk of second cancers in the female breast (HR: 0.67; 95\% CI: $0.52-$ $0.86 ; \mathrm{P}=0.002$ ). Age-adjusted competing-risk analysis showed generally consistent results.

Conclusions: Radiotherapy increased the risk of second cancers among cervical cancer patients. Those who underwent radiotherapy had a significantly higher risk of developing a second cancer in the colon, rectum and anus, lung and bronchus, corpus uteri, ovary, and urinary bladder.

Keywords: Cervical cancer; second malignancy; radiotherapy; competing-risk regression; propensity score matching analysis

Submitted Mar 23, 2021. Accepted for publication May 26, 2021.

doi: 10.21037/atm-21-1393

View this article at: https://dx.doi.org/10.21037/atm-21-1393 


\section{Introduction}

Cervical cancer is estimated to be the fourth most common cancer in women, with 570,000 cases and 311,000 deaths in 2018 (1). Radiotherapy can be used for the treatment of cervical cancer as a single modality or in combination with surgery or chemotherapy. However, a consensus has not been reached on the overall benefit of radiotherapy. Although radiotherapy has equivalent efficacy with surgery in the control of first cancer sites $(2,3)$, its side effects, especially the risk of second primary malignancy, have generated concerns about its use.

Cancer survivors are reported to be more predisposed to second primary malignancies (4). A study by Kleinerman et al. found that cervical cancer patients who underwent radiotherapy had a higher risk of developing second malignancies compared with the general population (5). Chaturvedi et al. confirmed in their study of 104,760 1 -year survivors that the risk of second malignancies was significantly higher at sites close to the cervix as a result of heavy irradiation (6). A previous study based on Surveillance, Epidemiology, and End Results (SEER) cancer registries suggested only a small proportion of second cancers were likely related to radiotherapy and that other factors including lifestyle and genetics had a greater influence (7).

As radiotherapy is an effective treatment modality, it is important to explore whether or not it increases the risk of second malignancies. This study performed competing-risk analysis using data from SEER to evaluate the impact of radiotherapy on the long-term risk of second malignancies. We present the following article in accordance with the STROBE reporting checklist (available at https://dx.doi. org/10.21037/atm-21-1393).

\section{Methods}

\section{Data source and cohort}

The National Cancer Institute's SEER program is an authoritative database for cancer statistics. This study performed statistical analysis on data from 9 registries including Atlanta, Connecticut, Detroit, Hawaii, Iowa, New Mexico, San Francisco-Oakland, Seattle-Puget Sound, and Utah. Cervical cancer patients aged minimum 20 years and diagnosed between January 1975 and November 2011 were selected for further screening. Those who met the following criteria were excluded: (I) patients whose cervical cancer was not considered the first primary cancer, (II) patients with incomplete survival and follow-up information, and
(III) patients who died within 5 years after the diagnosis of primary cervical cancer. The SEER registries used for analyses were last updated November 2016, and thus all enrolled patients had survived at least 5 years since diagnosis of the initial cervical cancer, revealing the longterm effect of radiation exposure on the incidence of second malignancies (8). The study was conducted in accordance with the Declaration of Helsinki (as revised in 2013).

\section{Study parameters}

Patient information was extracted from the database using SEER*Stat software 8.3.6. The following information was obtained from the database: year of diagnosis (1975-1984, 1985-1994, 1995-2004, or 2005-2011), age (20-39, $40-64$, or $\geq 65$ ), gender (male or female), ethnicity [White, Black, others (American Indian/Alaskan Native, Asian/ Pacific Islander), or unknown], histologic type (squamous, adenocarcinoma, or others), SEER cancer stage (localized, regional, distant, or unknown), histology (squamous cell carcinoma: codes 8070-8076 and 8084; adenocarcinoma: codes 8140, 8144, 8210, 8255, 8260-8263, 8310, 8323, 8384; or others), grade (well differentiated, moderately differentiated, poorly differentiated, undifferentiated, or unknown), whether a surgery was given (yes or no), whether chemotherapy was used (yes or no), and whether radiotherapy was used (yes or no).

\section{Statistical analysis}

SPSS version 25.0 (IBM Corporation, Armonk, NY, USA) and R software 3.6.3 (http://www.r-project.org) were used to perform statistical analysis. Statistical significance was achieved at a $\mathrm{P}$ value $<0.05$ ( 2 sides). Patient clinical information was recorded in the form of count or percentage, and Pearson's chi-square test was used to make comparisons.

Age-adjusted competing-risk regression analysis using the R package "cmprsk" was performed to assess the association between radiotherapy and second malignancies in order to avoid risk estimation bias caused by conventional statistical methods which improperly process data of patients who die before the event of interest, who do not experience the event before the end of follow-up, or who are lost to follow-up $(9,10)$. For the calculation of the whole risk of second malignancies, competing events were death and occurrence of second malignancies; for the calculation of the risk of single site second malignancies, competing 
events were death and other malignancies. Further, to ensure there were consistent distributions of clinical information for patients receiving radiotherapy and those not receiving radiotherapy, the competing-risk regression model was adjusted by propensity score matching (PSM). In the process of patient matching, each patient in one group was matched to a possible patient in the other group, with all unmatched patients excluded from the PSM-adjusted competing-risk regression model.

\section{Results}

\section{Patient information}

A total of 23,112 cervical cancer patients were identified for this study and enrolled for further analysis. Among them, $14,800(64.0 \%)$ patients received radiotherapy. Univariate analysis and multivariate logistic regression were applied to identify the difference between patients with radiotherapy and those without radiotherapy (Table 1). The proportion of cervical cancer patients receiving radiotherapy has increased since 1975 . Radiotherapy was more likely to be used in young patients [40-64 vs. 20-39: odds ratio (OR) 0.205, $95 \%$ confidence interval (CI): $0.183-0.229, \mathrm{P}<0.001 ; \geq 65$ vs. 20-39: OR: 0.526, 95\% CI: 0.476-0.582, $\mathrm{P}<0.001]$; black patients (OR: 5.828; 95\% CI: 2.742-12.389; $\mathrm{P}<0.001$ ); patients from other races (OR: 5.729; 95\% CI: $2.682-$ 12.236; $\mathrm{P}<0.001)$; patients of unknown race (OR: 7.600; 95\% CI: 3.551-16.266; $\mathrm{P}<0.001)$; patients histologically diagnosed as adenocarcinoma (OR: $1.547 ; 95 \%$ CI: 1.383-1.729; $\mathrm{P}<0.001$ ) or other types (OR: 1.306 ; 95\% CI: $1.131-1.508 ; \mathrm{P}<0.001)$; patients with regional invasion (OR: 1.025; 95\% CI: 0.871-1.207; $\mathrm{P}=0.765$ ), distant metastasis (OR: 7.397: 95\% CI: 6.226-8.788; $\mathrm{P}<0.001$ ), or unknown SEER stage (OR: 4.903; 95\% CI: 3.616-6.649; $\mathrm{P}<0.001$ ); patients with moderately differentiated cancer (OR: 1.637; 95\% CI: 1.428-1.876; $\mathrm{P}<0.001)$, poorly differentiated cancer (OR: 2.156; 95\% CI: 1.964-2.366; P<0.001), undifferentiated cancer (OR: 2.829; 95\% CI: 2.57-3.113; $\mathrm{P}<0.001$ ), or unknown grade (OR: 2.078 ; 95\% CI: 1.589 2.716; $\mathrm{P}<0.001)$; patients without surgery (OR: 2.910; 95\% CI: 2.497-3.392; $\mathrm{P}<0.001)$; and patients undergoing chemotherapy (yes vs. no/unknown: OR: 0.072; $95 \%$ CI: 0.062-0.084; $\mathrm{P}<0.001)$.

\section{Relation between radiotherapy and occurrence of second malignancies}

Out of a total of 23,112 cervical cancer patients, second malignancies were diagnosed in 2,545 (11.0\%) cases (Table 2). The top 12 most common sites of second malignancies were the lung and bronchus ( $\mathrm{N}=535,21.0 \%)$; female breast $(\mathrm{N}=522,20.5 \%)$; colon, rectum, and anus $(\mathrm{N}=347,13.6 \%)$; urinary bladder ( $\mathrm{N}=108,4.2 \%)$; lymphoma $(\mathrm{N}=108,4.2 \%)$; ovary $(\mathrm{N}=71,2.8 \%)$; vagina $(\mathrm{N}=67,2.6 \%)$; corpus uteri $(\mathrm{N}=58,2.3 \%)$; pancreas $(\mathrm{N}=57,2.2 \%)$; vulva $(\mathrm{N}=55,2.2 \%)$; stomach ( $\mathrm{N}=48,1.9 \%)$; and kidney (N=46, 1.8\%; Figure 1).

Competing-risk analysis revealed that radiotherapy significantly increased the risk of second malignancies [hazard ratio (HR): 1.62; 95\% CI: $1.50-1.75 ; \mathrm{P}<0.001$ ] and that it was significantly associated with higher risk of death (HR: 2.62; 95\% CI: 2.49-2.75; $\mathrm{P}<0.001$; Figure 2). The cumulative incidence for the top 12 most common second malignancies is shown in Figure 3.

Additionally, we analyzed changes in trends of the occurrence of second primary malignancies following the diagnosis of primary cervical cancer (Figure 4). The results demonstrated that the incidence of second malignancies declined as survival time increased among both populations of patients: those receiving radiotherapy (RT group) and those not receiving radiotherapy (no-RT group). Interestingly, the RT group started to show more second malignancies $10-15$ years after diagnosis of primary cervical cancer.

\section{Age-adjusted competing-risk analysis}

Generally, patients receiving radiotherapy had a higher risk of developing second malignancies (HR: 1.35; 95\% CI: 1.24-1.47; $\mathrm{P}<0.001$; Table 2). Compared with cervical cancer patients not receiving radiotherapy, those who received radiotherapy had a significantly higher risk of developing a second cancer in the small intestine (HR: 4.84; 95\% CI: 1.31-17.8; $\mathrm{P}=0.018$ ), lung and bronchus (HR: 1.35; 95\% CI: $1.24-1.47 ; \mathrm{P}<0.001$ ), corpus uteri (HR: 6.09; 95\% CI: 3.11-11.90; $\mathrm{P}<0.001$ ), ovary (HR: 2.61; 95\% CI: 1.52-4.49; $\mathrm{P}<0.001$ ), and urinary bladder (HR: 2.25 ; $95 \%$ CI: $1.47-$ $3.44 ; \mathrm{P}<0.001)$. However, radiotherapy significantly reduced the occurrence of second malignancies in the female breast (HR: 0.74; 95\% CI: 0.61-0.90; $\mathrm{P}=0.002$ ).

\section{PSM-adjusted competing-risk analysis}

Clinical information was further analyzed by PSM-adjusted competing-risk model using the nearest neighbor matching algorithm with a caliper of 0.1 . After matching with a ratio of 2 (patients receiving radiotherapy: patients not receiving 
Table 1 Univariate and multivariate analysis of patient information for factors that affected the application of radiotherapy

\begin{tabular}{|c|c|c|c|c|c|c|c|}
\hline Patient characteristics & $\begin{array}{c}\text { Total } \\
(\mathrm{N}=23,112)\end{array}$ & $\begin{array}{c}\text { No radiation } \\
(\mathrm{N}=14,800)\end{array}$ & $\begin{array}{l}\text { Radiation } \\
(\mathrm{N}=8,312)\end{array}$ & $P$ value & \multicolumn{3}{|c|}{ Logistic regression } \\
\hline \multicolumn{8}{|l|}{ Year of diagnosis } \\
\hline $1975-1984$ & $6,683(28.9 \%)$ & $4,075(27.5 \%)$ & $2,608(31.4 \%)$ & $<0.001$ & Reference & & \\
\hline 1985-1994 & $6,597(28.5 \%)$ & $4,381(29.6 \%)$ & $2,216(26.7 \%)$ & & 5.326 & $4.613-6.148$ & $<0.001$ \\
\hline 2005-2011 & $3,595(15.6 \%)$ & $2,197(14.8 \%)$ & $1,398(16.8 \%)$ & & 1.787 & $1.553-2.056$ & $<0.001$ \\
\hline \multicolumn{8}{|l|}{ Age, years } \\
\hline 20-39 & $9,144(39.6 \%)$ & 7,377 (49.8\%) & $1,767(21.3 \%)$ & $<0.001$ & Reference & & \\
\hline $40-64$ & $11,035(47.7 \%)$ & $6,234(42.1 \%)$ & $4,801(57.8 \%)$ & & .205 & $0.183-0.229$ & $<0.001$ \\
\hline Black & $3,010(13 \%)$ & $1,826(12.3 \%)$ & $1,184(14.2 \%)$ & & 5.828 & $2.742-12.389$ & $<0.001$ \\
\hline Others & $2,290(9.9 \%)$ & $1,295(8.8 \%)$ & $995(12 \%)$ & & 5.729 & $2.682-12.236$ & $<0.001$ \\
\hline Unknown & $213(0.9 \%)$ & $201(1.4 \%)$ & $12(0.1 \%)$ & & 7.600 & $3.551-16.266$ & $<0.001$ \\
\hline \multicolumn{8}{|l|}{ Histologic type } \\
\hline Squamous & 16,505 (71.4\%) & $10,052(67.9 \%)$ & $6,453(77.6 \%)$ & $<0.001$ & Reference & & \\
\hline Adenocarcinoma & $3,392(14.7 \%)$ & $2,415(16.3 \%)$ & $977(11.8 \%)$ & & 1.547 & $1.383-1.729$ & $<0.001$ \\
\hline Other & 3,215 (13.9\%) & 2,333 (15.8\%) & $882(10.6 \%)$ & & 1.306 & $1.131-1.508$ & $<0.001$ \\
\hline \multicolumn{8}{|l|}{ SEER stage } \\
\hline \multicolumn{8}{|l|}{ Grade } \\
\hline Well differentiated & $2,121(9.2 \%)$ & $1,524(10.3 \%)$ & $597(7.2 \%)$ & $<0.001$ & Reference & & \\
\hline Moderately differentiated & $5,211(22.5 \%)$ & $2,813(19 \%)$ & $2,398(28.8 \%)$ & & 1.637 & $1.428-1.876$ & $<0.001$ \\
\hline Poorly differentiated & $4,317(18.7 \%)$ & $1,911(12.9 \%)$ & $2,406(28.9 \%)$ & & 2.156 & $1.964-2.366$ & $<0.001$ \\
\hline Undifferentiated & $354(1.5 \%)$ & $170(1.1 \%)$ & $184(2.2 \%)$ & & 2.829 & $2.57-3.113$ & $<0.001$ \\
\hline Unknown & $11,109(48.1 \%)$ & $8,382(56.6 \%)$ & $2,727(32.8 \%)$ & & 2.078 & $1.589-2.716$ & $<0.001$ \\
\hline \multicolumn{8}{|l|}{ Surgery } \\
\hline Yes & $20,872(90.3 \%)$ & $14,248(96.3 \%)$ & 6,624 (79.7\%) & $<0.001$ & Reference & & \\
\hline No & $2,240(9.7 \%)$ & $552(3.7 \%)$ & $1,688(20.3 \%)$ & & 2.910 & $2.497-3.392$ & $<0.001$ \\
\hline \multicolumn{8}{|l|}{ Chemotherapy } \\
\hline Yes & $2,901(12.6 \%)$ & $329(2.2 \%)$ & $2,572(30.9 \%)$ & $<0.001$ & Reference & & \\
\hline No/unknown & $20,211(87.4 \%)$ & $14,471(97.8 \%)$ & $5,740(69.1 \%)$ & & 0.072 & $0.062-0.084$ & $<0.001$ \\
\hline
\end{tabular}

OR, odds ratio; $\mathrm{Cl}$, confidence interval; SEER, Surveillance, Epidemiology, and End Results. 
Table 2 The risk of second malignancies among cervical cancer patients after radiotherapy

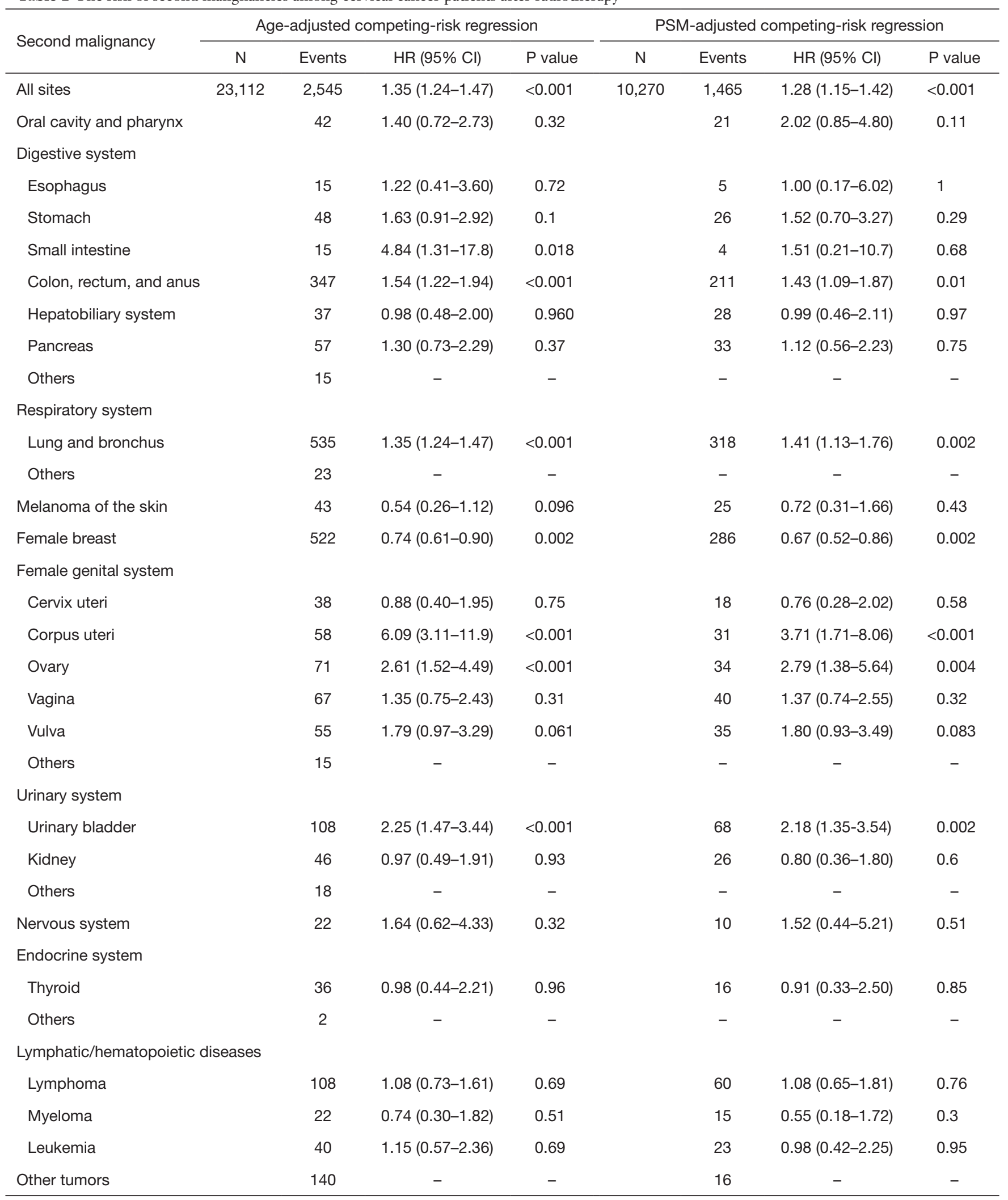

$\mathrm{HR}$, hazard ratio; $\mathrm{Cl}$, confidence interval; PSM, propensity score matching. 


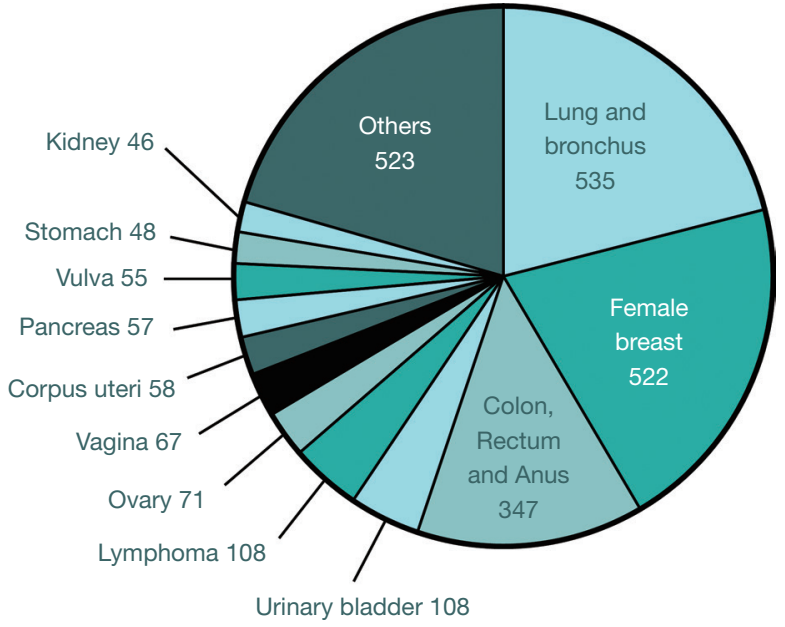

Figure 1 Sites of second malignancies after radiotherapy among cervical cancer patients.

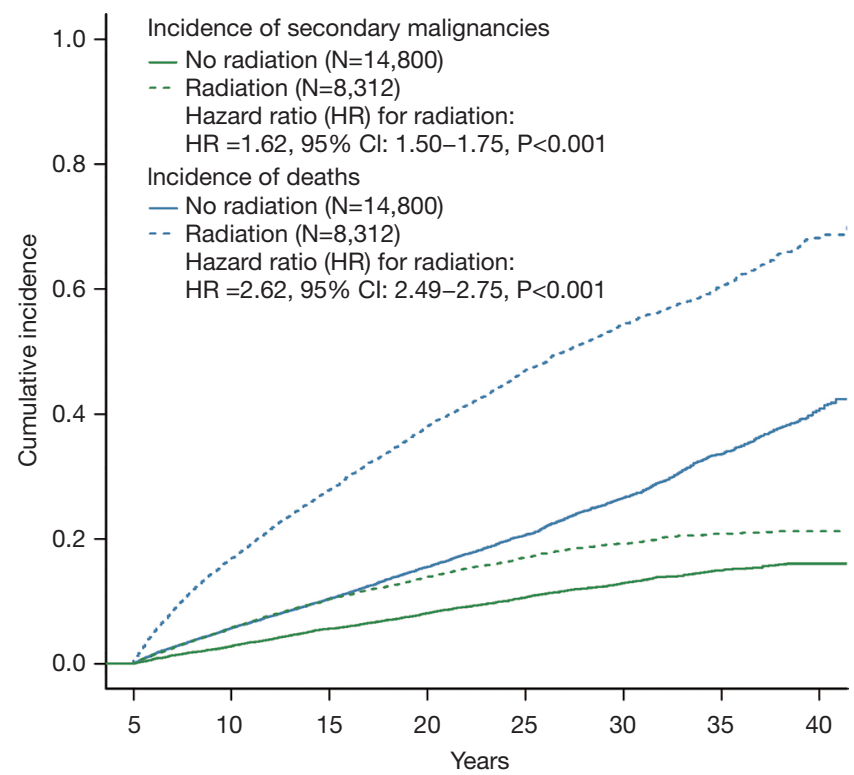

Figure 2 Cumulative incidence for the overall risk of second malignancies and death reported by age-adjusted competing-risk analysis.

radiotherapy $=2: 1), 10,270$ patients were included in the PSM-adjusted competing-risk analysis (Table 2). Patients receiving radiotherapy had a significantly higher risk of developing a second cancer in the colon, rectum, and anus (HR: 1.43; 95\% CI: 1.09-1.87; $\mathrm{P}=0.01$ ); lung and bronchus (HR: $1.41 ; 95 \%$ CI: $1.13-1.76 ; \mathrm{P}=0.002$ ); corpus uteri (HR: 3.71; 95\% CI: 1.71-8.06; P<0.001); ovary (HR: 2.79;
95\% CI: 1.38-5.64; $\mathrm{P}=0.004)$; and urinary bladder (HR: 2.18; 95\% CI: 1.35-3.54; $\mathrm{P}=0.002)$. However, radiotherapy significantly lowered the risk of a second cancer in the female breast (HR: 0.67; 95\% CI: 0.52-0.86; $\mathrm{P}=0.002$ ).

\section{Discussion}

This comprehensive, population-based study analyzed how radiotherapy affected the occurrence of second malignancies. The combined results from the unadjusted analysis and PSM-adjusted competing-risk analyses indicate that radiotherapy significantly increased the occurrence of a second cancer in the colon, rectum, and anus; lung and bronchus; corpus uteri; ovary; and urinary bladder; while a significant decrease in the risk of breast cancer was observed.

With the improvements in cancer prognoses in recent years, the occurrence of second malignancies is drawing increasingly more attention. Radiotherapy is an effective treatment modality for a variety of cancers. However, along with genetics, lifestyle, and chemotherapy, radiotherapy is considered a potential factor involved in increasing the risk of second malignancies (7). This is especially the case with cervical cancer. According to recent studies, the 5-year survival of all stages was up to $62.8 \%$ for all races in the United States between 2001 and 2009 (11). It has been estimated that half of cervical patients receive radiotherapy (12). Whether or not radiotherapy can lead to an increased risk of a second cancer has generated much concern. A systematic review and meta-analysis conducted in 2018 found an increased risk of rectal cancer after pelvic radiation for the treatment of cervical cancer [relative risk (RR) 1.61; 95\% CI: 1.10-2.35] (13). This finding was consistent with our results and those found in a number of previous studies $(5,14-17)$. It is generally believed that second cancers tend to occur in sites that have received radiation, so pelvic organs such as the rectum, corpus uteri, ovary, and urinary bladder could be more predisposed to second malignancies.

Our study found that lung cancer was the most common second malignancy in cervical cancer patients receiving radiotherapy and that radiotherapy significantly increased its risk (HR: 1.41; 95\% CI: 1.13-1.76; $\mathrm{P}=0.002$ ), which has been confirmed by other studies $(4,6)$. Notably, lung cancer has also been identified as the most common second cancer in survivors of bladder cancer; both bladder cancer and lung cancer are associated with smoking, which might cause somatic mutations (4). Even though the lung receives 

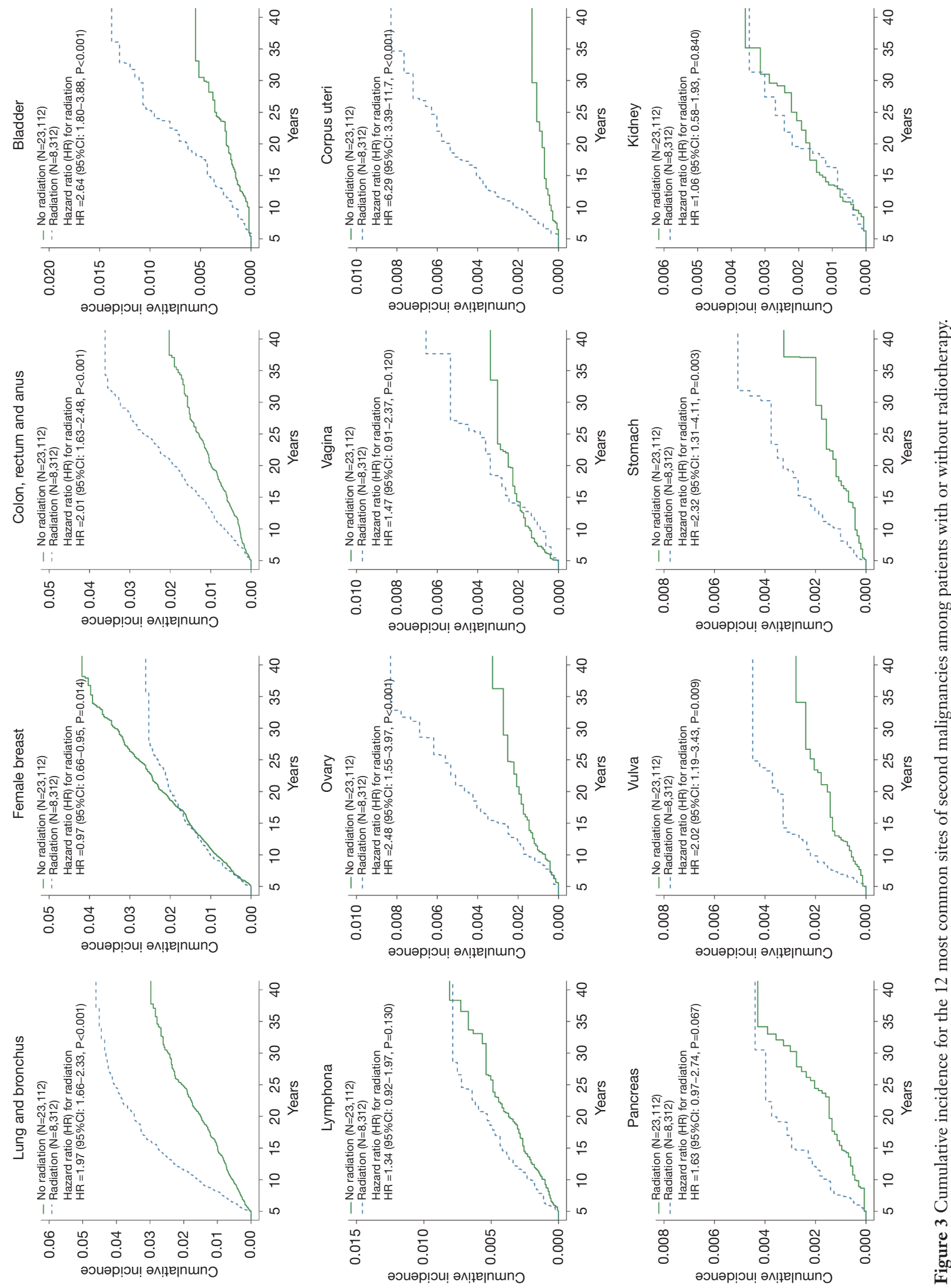


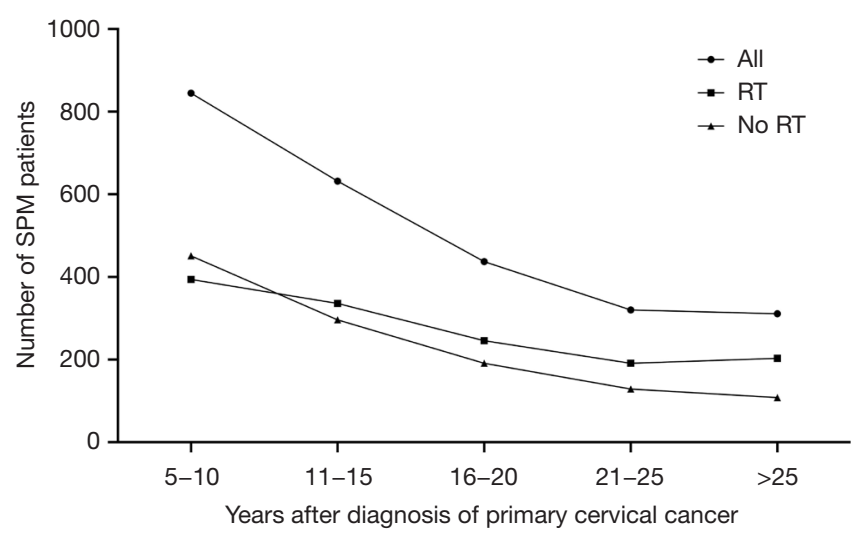

Figure 4 Changes in trends of the incidence of second primary malignancy (SPM) over years after diagnosis of first primary cervical cancer. RT, radiotherapy.

only a very small amount of radiation in the treatment of cervical cancer, smoking and radiation have been found to reinforce each other with respect to the development of subsequent cancers (18). A study by Arnold et al. found that cervical cancer patients who smoked and were receiving radiotherapy had an increased risk of a second tumor at smoking-related sites [incidence rate ratio (IRR): 1.6; 95\% CI: 1.2-2.3] (19). Unfortunately, one of the limitations of using the SEER database is that patient characteristics, such as smoking history, are unknown. However, previous population-based studies have provided evidence that smoking is associated with poor survival (20) and a higher risk of second malignancies $(19,21,22)$ in cervical cancer patients. As a result, the necessity of smoking cessation should be emphasized in cervical cancer patients, especially those receiving radiotherapy.

Human papillomavirus (HPV) infection is acknowledged as the main cause of cervical cancer and is also involved in the occurrence of second cancers. HPV-related sites include the cervix uteri, vulva, vagina, anus, and oropharynx (23). In this study, there was increased risk of a second cancer in the vulva (HR: 1.80 ; 95\% CI: 0.93-3.49), vagina (HR: 1.37; 95\% CI: 0.74-2.55), and oropharynx (HR: 2.02; 95\% CI: 0.85-4.80) among cervical cancer patients. However, the risk of a second tumor in the cervix uteri (HR: 0.76; 95\% CI: 0.28-2.02) was lower in cervical cancer patients than in the general population. The reasons for this remain to be further explored. According to a previous study, the risk of HPV-related second cancer varied by age and was especially high among cervical cancer survivors older than 70 years (19). This finding emphasizes the need for increasing patient education and enhanced screening in the future.

After analyzing changes in trends of the incidence of second malignancies over time, we found that incidence declined as survival time increased, which was observed in both the RT group and the no-RT group (Figure 4). The incidence rates were highest in the first 5 years $(5-10$ years since diagnosis of primary cervical cancer). However, the RT group started to show more second malignancies as time progressed (10-15 years after the diagnosis), which might indicate a long-term effect of radiotherapy on the incidence of second malignancies.

There are some limitations that should be addressed. First, the SEER database does not contain detailed patient information, such as smoking status, HPV infection status, or other environmental risk factors. Thus, this study was unable to analyze how these factors affect the occurrence of cervical cancer and related secondary malignancies. Second, this study did not consider other factors in the use of radiotherapy that could affect the occurrence of second malignancies. For example, intensity modulated radiotherapy (IMRT), a type of conformal radiotherapy, has been reported to be associated with increased risk of second cancer (24). The radiation dose might also affect this risk. These factors remain to be further investigated.

\section{Conclusions}

Radiotherapy appears to increase the risk of second cancers among cervical cancer patients. Our study found that those who underwent radiotherapy had a significantly higher risk of developing a second cancer in the colon, rectum, and anus; lung and bronchus; corpus uteri; ovary; and urinary bladder. Interestingly, radiotherapy seemed to be a protective factor for breast cancer in cervical cancer patients.

\section{Acknowledgments}

Funding: This study was funded by the Ministry of Science and Technology of the People's Republic of China (2016YFC0105207) and the National Key Research and Development Plan, the Ministry of Science and Technology of the People's Republic of China (2017YFC1311004).

\section{Footnote}

Reporting Checklist: The authors have completed the STROBE reporting checklist. Available at https://dx.doi. org/10.21037/atm-21-1393 
Conflicts of Interest: All authors have completed the ICMJE uniform disclosure form (available at https://dx.doi. org/10.21037/atm-21-1393). The authors have no conflicts of interest to declare.

Ethical Statement: The authors are accountable for all aspects of the work in ensuring that questions related to the accuracy or integrity of any part of the work are appropriately investigated and resolved. The study was conducted in accordance with the Declaration of Helsinki (as revised in 2013).

Open Access Statement: This is an Open Access article distributed in accordance with the Creative Commons Attribution-NonCommercial-NoDerivs 4.0 International License (CC BY-NC-ND 4.0), which permits the noncommercial replication and distribution of the article with the strict proviso that no changes or edits are made and the original work is properly cited (including links to both the formal publication through the relevant DOI and the license). See: https://creativecommons.org/licenses/by-nc-nd/4.0/.

\section{References}

1. Arbyn M, Weiderpass E, Bruni L, et al. Estimates of incidence and mortality of cervical cancer in 2018: a worldwide analysis. Lancet Glob Health 2020;8:e191-203.

2. Landoni F, Maneo A, Colombo A, et al. Randomised study of radical surgery versus radiotherapy for stage Ib-IIa cervical cancer. Lancet 1997;350:535-40.

3. Garg G, Shah JP, Toy EP, et al. Stage IIA1 versus stage IIA2 cervical cancer: does the new staging criteria predict survival? Int J Gynecol Cancer 2011;21:711-6.

4. Donin N, Filson C, Drakaki A, et al. Risk of second primary malignancies among cancer survivors in the United States, 1992 through 2008. Cancer 2016;122:3075-86.

5. Kleinerman RA, Boice JD Jr, Storm HH, et al. Second primary cancer after treatment for cervical cancer. An international cancer registries study. Cancer 1995;76:442-52.

6. Chaturvedi AK, Engels EA, Gilbert ES, et al. Second cancers among 104,760 survivors of cervical cancer: evaluation of long-term risk. J Natl Cancer Inst 2007;99:1634-43.

7. Berrington de Gonzalez A, Curtis RE, Kry SF, et al. Proportion of second cancers attributable to radiotherapy treatment in adults: a cohort study in the US SEER cancer registries. Lancet Oncol 2011;12:353-60.
8. Preston DL, Ron E, Tokuoka S, et al. Solid cancer incidence in atomic bomb survivors: 1958-1998. Radiat Res 2007;168:1-64.

9. Scrucca L, Santucci A, Aversa F. Competing risk analysis using R: an easy guide for clinicians. Bone Marrow Transplant 2007;40:381-7.

10. Fine JP, Gray RJ. A Proportional Hazards Model for the Subdistribution of a Competing Risk. J Am Stat Assoc 1999;94:496-509.

11. Benard VB, Watson $M$, Saraiya $M$, et al. Cervical cancer survival in the United States by race and stage (2001-2009): Findings from the CONCORD-2 study. Cancer 2017;123 Suppl 24:5119-37.

12. Kleinerman R, Kosary C, Hildesheim A. New malignancies among cancer survivors: SEER cancer registries, 19732000. National Cancer Institute 2006:207-29.

13. Rombouts AJM, Hugen N, van Beek JJP, et al. Does pelvic radiation increase rectal cancer incidence? - A systematic review and meta-analysis. Cancer Treat Rev 2018;68:136-44.

14. Clarke EA, Kreiger N, Spengler RF. Second primary cancer following treatment for cervical cancer. Can Med Assoc J 1984;131:553-6.

15. Boice JD Jr, Day NE, Andersen A, et al. Second cancers following radiation treatment for cervical cancer. An international collaboration among cancer registries. J Natl Cancer Inst 1985;74:955-75.

16. Hiyama T, Fujimoto I, Hanai A, et al. Occurrence of second primary cancers among patients with cervical cancer in Osaka, Japan. National Cancer Institute Monograph 1985;MONOGR. 69:181-4.

17. Pettersson F, Fotiou S, Einhorn N, et al. Cohort study of the long-term effect of irradiation for carcinoma of the uterine cervix. Second primary malignancies in the pelvic organs in women irradiated for cervical carcinoma at Radiumhemmet 1914-1965. Acta Radiol Oncol 1985;24:145-51.

18. Travis LB, Gospodarowicz M, Curtis RE, et al. Lung cancer following chemotherapy and radiotherapy for Hodgkin's disease. J Natl Cancer Inst 2002;94:182-92.

19. Arnold M, Liu L, Kenter GG, et al. Second primary cancers in survivors of cervical cancer in The Netherlands: Implications for prevention and surveillance. Radiother Oncol 2014;111:374-81.

20. Coker AL, DeSimone CP, Eggleston KS, et al. Smoking and survival among Kentucky women diagnosed with invasive cervical cancer: 1995-2005. Gynecol Oncol 2009;112:365-9. 
21. Hemminki K, Dong C, Vaittinen P. Second primary cancer after in situ and invasive cervical cancer. Epidemiology 2000;11:457-61.

22. Karahalios E, English D, Thursfield V, et al. Second primary cancers in Victoria. Melbourne: Cancer Council Victoria 2009;113.

23. de Martel C, Ferlay J, Franceschi S, et al. Global burden of cancers attributable to infections in 2008: a review and synthetic analysis. Lancet Oncol 2012;13:607-15.

24. Hall EJ. Intensity-modulated radiation therapy, protons, and the risk of second cancers. Int J Radiat Oncol Biol Phys 2006;65:1-7.

(English Language Editors: A. Muijlwijk and J. Gray)
Cite this article as: Wu Y, Chong Y, Han C, Kang K, Liu Z, Zhang F. Second primary malignancies associated with radiation therapy in cervical cancer patients diagnosed between 1975 and 2011: a population-based competing-risk study. Ann Transl Med 2021;9(17):1375. doi: 10.21037/atm-21-1393 